\title{
Efficacy and safety of Qing-Feng-Gan-Ke Granules in patients with postinfectious cough: study protocol of a novel-design phase III placebo-controlled, double-blind randomized trial
}

Wei Liu ${ }^{1 \dagger}$, Hongli Jiang ${ }^{1 \dagger}$, Ruiming Zhang ${ }^{1}$, Faguang Jin², Liangji Liư ${ }^{3}$, Youyu Long ${ }^{4}$, Liying Cui ${ }^{5}$, Suyun Li ${ }^{6}$, Yunqing Zhong ${ }^{7}$ and Bing Mao ${ }^{1 *}$

\begin{abstract}
Background: Postinfectious cough (PIC) is a common condition that affects millions of people worldwide every year. There is Western medicine for this condition but the treatment effect is often incomplete. Traditional Chinese medicine (TCM) has been increasingly prescribed for patients with PIC. Preliminary trials on Qing-Feng-Gan-Ke-Granules (QFGKG) conveyed promising results in treating PIC. This protocol describes an ongoing phase III randomized controlled clinical trial, designed according to a novel methodology of "one study, one primary outcome", with the objective of evaluating the efficacy and safety of QFGKG in patients suffering from PIC.
\end{abstract}

Methods/Design: This is a multicenter, phase III, randomized, double-blind, parallel-group, placebo-controlled clinical trial, comprising two simultaneously conducted study parts, part A and part B, intending to investigate two primary outcomes, i.e. time to cough resolution and cough symptom score, respectively. A total of 480 patients, aged 18 to 65 years, who complain of an ongoing persistent cough that has been lasting $\geq 3$ weeks, will be recruited from six participating sites and then randomized to receive QFGKG $12.0 \mathrm{~g}$ twice daily or placebo $12.0 \mathrm{~g}$ twice daily. Each part will enroll 240 patients, with 180 patients being allocated to the QFGKG group and 60 to the placebo group.

Discussion: Although traditional Chinese medicine is a structured intervention that has shown some promise in treating persistent cough, existing unconvincing evidence has noted limitations. This is a rare well-designed and rigorously-controlled, randomized, double-blind trial to evaluate the effects and safety of a Chinese herbal medicine in patients with postinfectious cough, providing tangible benefits for clinical research. Results of this trial are inclined to be conjectured as more truthful by implementing separate study parts that specifically estimate exclusive primary outcome. It will not only provide robust clinical evidence on the efficacy and safety of QFGKG for postinfectious cough, but will also provide a critical piece of information on the availability and superiority of a novel methodology for future clinical trials. The current trial is ongoing with recruitment of the predetermined number of patients being in progress.

Trial registration: The two parts of this trial were separately registered with the Chinese Clinical Trial Registry: ChiCTR-TRC-13003278 (part A); and ChiCTR-TRC-13003337 (part B).

\footnotetext{
*Correspondence: maobing2013@yeah.net

${ }^{\dagger}$ Equal contributors

'Department of Integrated Traditional and Western Medicine, West China Hospital of Sichuan University, 37 Guoxue Lane, Chengdu, Sichuan Province 610041, China

Full list of author information is available at the end of the article
}

\section{Biomed Central}

(c) 2015 Liu et al. Open Access This article is distributed under the terms of the Creative Commons Attribution 4.0 International License (http://creativecommons.org/licenses/by/4.0/), which permits unrestricted use, distribution, and reproduction in any medium, provided you give appropriate credit to the original author(s) and the source, provide a link to the Creative Commons license, and indicate if changes were made. The Creative Commons Public Domain Dedication waiver (http://creativecommons.org/publicdomain/zero/1.0/) applies to the data made available in this article, unless otherwise stated. 


\section{Background}

Cough is the most common symptom presenting to primary healthcare services. Postinfectious cough (PIC) is considered as one of the top causes of subacute cough, which occurs in 11 to $25 \%$ of patients with upper respiratory tract infection (URTI) $[1,2]$. It involves a persistent cough lasting $\geq 3$ weeks or even months [3] (but normally $<8$ weeks) secondary to the acute symptoms of a common cold or upper respiratory tract infection (URTI) [4, 5]. Although PIC is always self-limited and is not associated with severe debilitation or mortality, persistent cough may impair the quality of daily life [4]. Therefore, many patients with PIC are frequently impelled to seek medical help [6].

Aside from pertussis, however, despite the fact that many pathogenic agents including respiratory viruses (particularly respiratory syncytial virus, adenoviruses, parainfluenza, and influenza), M pneumoniae and Chlamydophila pneumoniae have been implicated in children $[4,5,7]$, the role of infectious aetiology in PIC in adults is rarely confirmed till now [8]. Poor knowledge on the pathogenesis of PIC, which is supposed to be frequently multifactorial [2, 8-10], results in a diagnostic challenge for physicians. Patients are constantly investigated for other causes of persistent cough [6]. Therapy with antibiotics has no role, as there is no evidence that bacterial infection plays a role in adult patients $[4,5]$. Cysteinyl leukotrienes were considered to be involved in the pathogenesis of PIC in recent studies, however, montelukast failed to effectively treat PIC in a latest randomized trial [11], leading to a controversy on its role in PIC. Therefore, PIC can be a troublesome problem for physicians and patients alike [6]. Currently available therapies, including inhaled ipratropium, inhaled or oral corticosteroids, central acting antitussive agents such as codeine and dextromethorphan, can only be used for symptom relief $[4-7,12,13]$. They have not been proved to be effective in reducing the cough duration or improving the quality of life [4]. And above all, a range of adverse outcomes including blurred vision, confusion, difficulty in urination, drowsiness or dizziness, body rash/itching, nausea or vomiting, constipation, diarrhea, sedation, nervousness are elicited occasionally [14-16]. For these reasons, complementary and alternative medicine is becoming more and more popular in patients with PIC, such as a combination of honey and coffee [6] as well as traditional herbal medicines.

The use of traditional Chinese medicine (TCM) as a complementary and alternative therapy for cough can be dated back to thousands of years ago. People in China who suffer from a persistent cough always give first priority to TCM. With an increasing number of TCM formulas developed for clinical use, an overview to facilitate the discussion of its pros and cons is warranted. A recent comprehensive systematic review of randomized controlled trials demonstrated potential curative effects and reassured the safety of TCM in the management of PIC [17]. Unfortunately, due to the undesirable quality of included studies, the definite effect of TCM turned to be inconclusive in this review. Therefore, a rigorously designed randomized trial becomes imperative.

TCM offers a holistic vision on disease, and syndrome differentiation is the cornerstone of TCM prescription. Differentiation means comprehensive analysis, and syndrome refers to symptoms and signs. Differentiation of syndromes implies that the patient's symptoms and signs collected by the four diagnostic methods (inspection, auscultation ant olfaction, inquiry, pulse-taking and palpation) are analyzed and summarized to identify the etiology, nature and location of a disease, thereby determining what syndrome the disease belongs to. Based on both clinical experience and literature review, "pathogenic-wind invading lung syndrome" is considered as the most common TCM syndrome in patients with PIC [17]. TCM patent drug Qing-Feng-Gan-Ke-Granules (QFGKG), designed on the basis of a classic formula, comprises four widely used herbal plants (Ephedra sinica Stapf, Sinomenium acutum, Stemona japonica (Blume) Miq., and Aster tataricus L.f.). It has been shown that QFGKG is very effective in dispelling wind and relieving cough and is specifically appropriate for treating "pathogenic-wind invading lung syndrome". Unpublished preliminary phpartacodynamics studies in rats showed that QFGKG could significantly alleviate sulfur dioxide-induced cough and ammonium hydroxide-induced cough, increase the tracheal phenolsulfonphthalein excretion, improve the peritoneal capillary permeability reduced by acetic acid, relieve asthma caused by acetyl choline and histamine inhalation, inhibit carrageenin-induced toes swelling, suppress dinitrochlorobenzene-induced delayed type hypersensitivity, which suggests its antitussive, anti-inflammatory and immunosuppressive properties. Subsequent clinical studies investigated its efficacy and feasibility in individuals with PIC $[18,19]$. In addition, a previous multi-center phase II trial [20] investigated the dose-effect relationship and dosesafety relationship of QFGKG, with primary outcomes of time to cough relief and time to cough resolution, as well as secondary outcomes of cough symptom score, cough recovery rate, TCM curative effect, and Chinese version of cough-specific quality of life questionnaire (CQLQ) score [21]. In this phase II trial, 180 patients were enrolled and randomly assigned to receive high dose of QFGKG (12.0 g, twice daily), or low dose of QFGKG (6.0 g, twice daily) plus dummy QFGKG (6.0 g, twice daily), or dummy QFGKG (12.0 g, twice daily) alone. Great improvements in all outcomes, especially the time to cough resolution and cough symptom score, were observed in the high dose group. To the best of our knowledge, the phase II study was the first well-designed placebo-controlled clinical trial that reported TCM as a remedy for PIC, and its resultful 
conclusions provide foundations for the current phase III trial.

"One study, one primary outcome", a novel methodology for clinical trial design, was published by China Food and Drug Administration (CFDA). The core of this concept lies in the supposition that study results will be more credible if the whole study is single-mindedly and exclusively designed for the unique outcome of main interest. Moreover, the SPIRIT guidance suggests a minimum number of primary outcomes in clinical trials because multiple primary outcomes can introduce problems with multiplicity, selective reporting, and interpretation when there are inconsistent results across outcomes [22]. Thus, although this design has not been widely implemented in clinical trials, we prefer to conduct a tentative trial in an attempt to provide some practical information.

The objective of this trial is to demonstrate the superior efficacy and safe of QFGKG over placebo in adult individuals with PIC by addressing the following hypotheses: (1) That QFGKG will significantly shorten the cough duration in patients with PIC; (2) That QFGKG will significantly relieve the main clinical symptoms in patients with PIC; (3) That QFGKG will increase the quality of life in patients with PIC; (4) That QFGKG is safe and will not result in any serious side effects; (5) That the novel design methodology of "one study, one primary outcome" is feasible and desirable in clinical trials.

\section{Methods/Design \\ Study designs}

This trial is designed as a randomized, double-blind, placebo-controlled, parallel, phase III, superiority trial, with allocations stratified by participating centers and randomization performed as block at a ratio of 3:1, adhering to the recommendation of "CONSORT statement" [23] and "SPIRIT statement" [24]. As to the allocation ratio, generally speaking, for a superiority trial using placebo as a control intervention, 3:1 is recommended by Provisions for Drug Registration (http://www.sda.gov.cn/ $\mathrm{ypzcgl} / \mathrm{ml} . \mathrm{htm})$, taking into full consideration of statistical significance, ethical requirement and risk-cost relationship. This trial consists of two simultaneously conducted study parts (part A and part B), currently involving six participating centers across China: West China Hospital of Sichuan University, Tangdu hospital of The Fourth Military Medical University, Affiliated Hospital of Jiangxi University of TCM, the Second Affiliated Hospital of Tianjin University of TCM, Affiliated Hospital of Inner Mongolia Medical University, and the First Affiliated Hospital of Henan University of TCM. These centers were defined primarily geographically. In each participating center, a chief investigator will be appointed to be responsible for the whole clinical process (subject recruitment, intervention, follow-up, and data collection), adhering to the study protocol. All personnel involved in this trial will be trained prior to trial initiation. Training program is about study protocol and relevant skills, especially the skill of TCM syndrome differentiation. Staff placement and individual responsibility will also be elaborated in the training session. Besides, investigator's brochure developed by the sponsor to facilitate the smooth running of the trial will also be provided. This protocol is designed largely depending on the results of previous phase II trial. Both study parts have been registered with Chinese Clinical Trial Registry (http://www.chictr.org). This protocol has two amendments. The schematic diagram and

Table 1 Schematic diagram of part A

\begin{tabular}{|c|c|c|c|c|}
\hline \multirow{2}{*}{$\begin{array}{l}\text { Phase } \\
\text { Time-point }\end{array}$} & \multicolumn{4}{|c|}{ Supervision period } \\
\hline & Baseline & Visit 1 & Visit 2 & Visit 3 \\
\hline Day & 0 & $8 \pm 1$ & $15 \pm 2$ & Follow-up \\
\hline Basic history collection & $\times$ & & & \\
\hline Informed consent & $\times$ & & & \\
\hline Demographics & $\times$ & & & \\
\hline $\begin{array}{l}\text { Enrollment based on the } \\
\text { inclusion/exclusion criteria }\end{array}$ & $\times$ & & & \\
\hline $\begin{array}{l}\text { Coexisting diseases or } \\
\text { symptoms }\end{array}$ & $x$ & & & \\
\hline Concomitant treatments & & $x$ & $\times$ & \\
\hline \multicolumn{5}{|l|}{ Efficacy evaluation } \\
\hline Cough symptom score & $\times$ & $x$ & $\times$ & $x$ \\
\hline TCM syndrome score & $\times$ & $x$ & $\times$ & \\
\hline \multicolumn{5}{|l|}{ Safety evaluation } \\
\hline Vital signs & $\times$ & $x$ & $\times$ & \\
\hline $\begin{array}{l}\text { Blood routine and urine } \\
\text { routine }\end{array}$ & $\times$ & & $\times$ & \\
\hline $\begin{array}{l}\text { Liver function (ALT, AST, STB, } \\
\text { ALP, } \gamma-G T \text { ) }\end{array}$ & $\times$ & & $\times$ & \\
\hline Renal function (Cr, BUN) & $\times$ & & $\times$ & \\
\hline ECG & $\times$ & & $\times$ & \\
\hline Adverse events & & $\times$ & $\times$ & \\
\hline \multicolumn{5}{|l|}{ Screening tests } \\
\hline$C R$ & $\times$ & & & \\
\hline BPT & $\times$ & & & \\
\hline $\begin{array}{l}\text { UPT (women of childbearing } \\
\text { age) }\end{array}$ & $\times$ & & & \\
\hline \multicolumn{5}{|l|}{ Other steps } \\
\hline Randomization allocation & $\times$ & & & \\
\hline Drug distribution & $\times$ & & & \\
\hline Remaining drug count & & & $\times$ & \\
\hline $\begin{array}{l}\text { Patient record card distribution/ } \\
\text { recycling }\end{array}$ & $\times$ & & $\times$ & \\
\hline
\end{tabular}


Table 2 Schematic diagram of part B

\begin{tabular}{|c|c|c|c|c|}
\hline \multirow{2}{*}{$\begin{array}{l}\text { Phase } \\
\text { Time-point }\end{array}$} & \multicolumn{4}{|c|}{ Supervision period } \\
\hline & Baseline & Visit 1 & Visit 2 & Visit 3 \\
\hline Day & 0 & $6 \pm 1$ & $11 \pm 1$ & Follow-up \\
\hline Basic history collection & $x$ & & & \\
\hline Informed consent & $\times$ & & & \\
\hline Demographics & $\times$ & & & \\
\hline $\begin{array}{l}\text { Enrollment based on the } \\
\text { inclusion/exclusion criteria }\end{array}$ & $x$ & & & \\
\hline $\begin{array}{l}\text { Coexisting diseases or } \\
\text { symptoms }\end{array}$ & $x$ & & & \\
\hline Concomitant treatments & & $x$ & $\times$ & \\
\hline \multicolumn{5}{|l|}{ Efficacy evaluation } \\
\hline Cough symptom score & $\times$ & $\times$ & $\times$ & $\times$ \\
\hline Cough VAS score & $\times$ & $x$ & $\times$ & \\
\hline TCM syndrome score & $x$ & $x$ & $\times$ & \\
\hline CQLQ & $\times$ & & $\times$ & \\
\hline \multicolumn{5}{|l|}{ Safety evaluation } \\
\hline Vital signs & $x$ & $x$ & $\times$ & \\
\hline $\begin{array}{l}\text { Blood routine and urine } \\
\text { routine }\end{array}$ & $\times$ & & $\times$ & \\
\hline $\begin{array}{l}\text { Liver function (ALT, AST, STB, } \\
\text { ALP, } Y \text {-GT) }\end{array}$ & $x$ & & $x$ & \\
\hline Renal function (Cr, BUN) & $\times$ & & $\times$ & \\
\hline ECG & $\times$ & & $\times$ & \\
\hline Adverse events & & $x$ & $\times$ & \\
\hline \multicolumn{5}{|l|}{ Screening tests } \\
\hline$C R$ & $\times$ & & & \\
\hline BPT & $\times$ & & & \\
\hline $\begin{array}{l}\text { UPT (women of childbearing } \\
\text { age) }\end{array}$ & $\times$ & & & \\
\hline \multicolumn{5}{|l|}{ Other steps } \\
\hline Randomization allocation & $\times$ & & & \\
\hline Drug distribution & $x$ & & & \\
\hline Remaining drug count & & & $\times$ & \\
\hline $\begin{array}{l}\text { Patient record card distribution/ } \\
\text { recycling }\end{array}$ & $\times$ & & $\times$ & \\
\hline
\end{tabular}

flow chart of study process are shown in Tables 1, 2 and Fig. 1.

\section{Study participants}

A total of 480 patients will be recruited and averagely assigned to each part. Each center will recruit an equal number of patients; 60 patients will be recruited at each individual center in part $\mathrm{A}$ and 80 patients will be enrolled at each individual center in part B. Patients, who are diagnosed as having PIC in one of the participating centers according to the guideline issued by the Chinese Medical

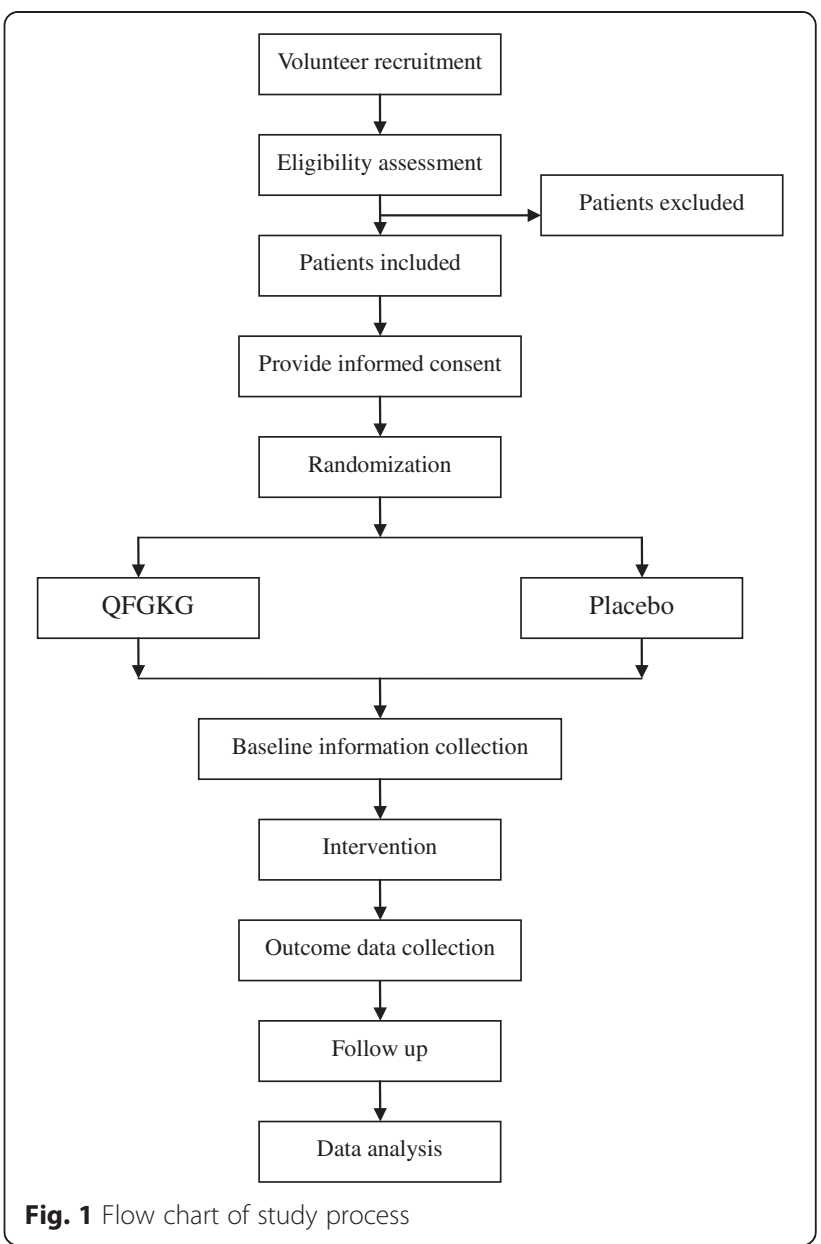

Association [25], aged between 18 and 65 years, with a TCM syndrome being differentiated as "pathogenic-wind invading lung" based on the Guidelines for Clinical Research of New Chinese Medicine [26], are eligible for enrollment. Diagnosis criteria of PIC are presented in Table 3. Inclusion and exclusion criteria are listed in Table 4, and TCM syndrome diagnostic criteria, based on State Administration of Traditional Chinese Medicine

Table 3 Diagnosis criteria for postinfectious cough

- Cough that has been present following symptoms of an upper respiratory tract infection;

- Irritable dry cough or cough with small amounts of white mucus;

- Cough lasts for at least 3 weeks, but normally no longer than 8 weeks;

- Negative findings of chest radiograph;

- Negative results of bronchial provocation test and dilation test;

- Exclude other causes which could result in cough, such as upper airway cough syndrome(UACS), cough variant asthma(CVA), eosinophilic bronchitis(EB) and gastroesohageal reflux disease(GERC);

- Exclude severe pulmonary diseases including chronic obstructive pulmonary disease(COPD),lung cancer, pulmonary tuberculosis, et al.;

- Exclude hypertension that involves use of angiotensin converting enzyme inhibitor(ACEI) currently. 
Table 4 Selection criteria for eligible patients

\begin{tabular}{|c|c|}
\hline $\begin{array}{l}\text { - Inclusion criteria for study part } \\
\text { A }\end{array}$ & - Inclusion criteria for study part B \\
\hline - Postinfectious cough & - Postinfectious cough \\
\hline $\begin{array}{l}\text { - Syndrome of "pathogenic-wind } \\
\text { invading lung" }\end{array}$ & $\begin{array}{l}\text { - Syndrome of "pathogenic-wind } \\
\text { invading lung" }\end{array}$ \\
\hline $\begin{array}{l}\text { - Daytime cough symptom score } \\
\geq 2 \text { and/or nighttime cough } \\
\text { symptom score } \geq 2\end{array}$ & - Total cough symptom score $\geq 4$ \\
\hline $\begin{array}{l}\text { - Persistent cough } \geq 3 \text { weeks } \\
\text { and } \leq 6 \text { weeks }\end{array}$ & $\begin{array}{l}\text { - Persistent cough } \geq 3 \text { weeks and } \leq \\
6 \text { weeks }\end{array}$ \\
\hline - Age $18-65$ years old & - Age 18-65 years old \\
\hline $\begin{array}{l}\text { - Agreement to participate and } \\
\text { provision of informed consent }\end{array}$ & $\begin{array}{l}\text { - Agreement to participate and } \\
\text { provision of informed consent }\end{array}$ \\
\hline \multicolumn{2}{|l|}{ - Exclusion criteria } \\
\hline \multicolumn{2}{|l|}{ - Body temperature $>37.3^{\circ} \mathrm{C}$} \\
\hline \multicolumn{2}{|l|}{$\begin{array}{l}\text { - Use of any medication intended } \\
\text { to treat PIC within } \\
\text { one week before enrollment }\end{array}$} \\
\hline \multicolumn{2}{|l|}{$\begin{array}{l}\text { - } \text { ALT/AST }>1.5 \text { times than the } \\
\text { upper limit of normal } \\
\text { reference values; Abnormal } \\
\text { blood creatinine; Urine protein } \\
\text { qualitative test }>+ \text {; } \\
\text { Blood leukocyte count } \\
<3.0 \times 10^{9} / \mathrm{L} \text { or }>10.0 \times 10^{9} / \mathrm{L} ; \\
\text { and/or neutrophil percentage }>80 \%\end{array}$} \\
\hline \multicolumn{2}{|l|}{$\begin{array}{l}\text { - History of any severe underlying } \\
\text { diseases } \\
\text { (e.g. cardiovascular, hematological, } \\
\text { hepatic, renal); or other } \\
\text { life-threatening disabling } \\
\text { conditions and concomitant } \\
\text { diseases (e.g. cancer, AIDS) }\end{array}$} \\
\hline \multicolumn{2}{|l|}{$\begin{array}{l}\text { - Pregnancy or potential pregnancy } \\
\text { or lactation }\end{array}$} \\
\hline \multicolumn{2}{|l|}{$\begin{array}{l}\text { - Hypersensitivity or allergy to any } \\
\text { component of the test drug }\end{array}$} \\
\hline \multicolumn{2}{|l|}{$\begin{array}{l}\text { - Current psychiatric disorders or } \\
\text { legal disability }\end{array}$} \\
\hline \multicolumn{2}{|l|}{$\begin{array}{l}\text { - Current or previous admission } \\
\text { to other investigational } \\
\text { drug studies within } \\
3 \text { months before enrollment }\end{array}$} \\
\hline $\begin{array}{l}\text { - Inability to complete the trial } \\
\text { as decided by the investigators }\end{array}$ & \\
\hline
\end{tabular}

guidelines [27], are elaborated in Table 5. Some discrepancies should be noticed in the inclusion criteria of two parts. Part A is designed to focus on the time when cough can be resolved completely, thus patients' cough severity is relatively mild in order to yield more expected results. On the other hand, part B is designed to calculate the dynamic change in cough symptom score across four time-points (see Table 2), so the cough severity of patients recruited in this part is relatively severe for more obvious changes.

Patients must provide written informed consent before any study procedures occur. Participants would
Table 5 Diagnostic criteria for TCM differentiation of "pathogenic-wind invading lung syndrome"

\begin{tabular}{ll}
\hline Item & Symptoms and signs \\
\hline *Primary symptom & persistent cough \\
Secondary symptoms & 1) *throat itching, cough sensitivity to \\
& cold air, heat air or strange odors and/or; \\
& 2) sticky and scanty sputum, dry throat \\
& and chest congestion \\
*Tongue picture & slight red tongue proper with/without \\
& red tip; thin white tongue coating \\
& normal or floating pulse
\end{tabular}

* Items which must be satisfied for a diagnosis of TCM differentiation of "pathogenic-wind invading lung syndrome"

be excluded if they have any of the following conditions: 1 ) misdiagnosis; 2) poor drug compliance (taking $<80 \%$ or $>$ $120 \%$ of the required dosage); 3 ) inability to complete the follow-up; 4) aggravations (such as dyspnea) that happen within $24 \mathrm{~h}$ after taking the test drug (on this occasion, patients will be treated with conventional therapies and considered as an invalid case, whose last data would be collected in Full Analysis Set (FAS)); 5) hypersensitivity or other severe adverse reactions; and 6) voluntary withdrawal. The trial will be terminated prior to its planned end date in the context of the following accidents: 1 ) protocol deviations; 2) inadvisable or flawed protocol; 3) compelling suspension forced by authoritative agencies; and 4) discontinuation decided by the trial sponsor (managerial or financial difficulties).

\section{Randomization and blinding}

Eligible patients will be randomized in a ratio of 3:1 to QFGKG group or placebo group, with stratification by participating center. A centralized randomization will be employed. The random coding of each center as well as the allocation sequence are generated electronically using SAS 6.12 software. Assignments are concealed in sequentially prepared coded, sealed, opaque envelopes, in which the corresponding intervention group of the randomization list will be found. A professional statistician who is independent of intervention and data collection is appointed to choose appropriate block length to ensure concealment. A group of research assistants who are not involved in recruitment, intervention or assessment of outcomes will prepare the envelopes. Staff responsible for recruitment is not allowed to take part in the group allocation. Only after the former participant is enrolled and his/her baseline testing is completed, the next envelope could be opened by researchers responsible for allocation. Participants will be randomized to the intervention group or the control group in line with numbered envelope under instructions from drug managers at each center. Blind codes along with random seeds and block length will be recorded in duplicate and delivered to Good Clinical Practice (GCP) Centre 
of West China Hospital and the sponsor for safekeeping. Patients and all engaged personnel including the sponsor remain blind to allocation and intervention throughout the whole process. Placebo will be packaged with imitative similarity in appearance, smell and taste with QFGKG. However, personnel who are designated to prepare drug packaging and labeling will not be blinded. The prepared emergency code-break envelope that contains allocation information, and emergency measures as well as contact details of the responsible department will be distributed to study centers along with drugs. Patients reported side effects will be recorded in the case report forms (CRFs) detailedly by physicians at visit 1 and visit 2 . If an unpredictable severe adverse event happens, the chief investigator is qualified to open the emergency envelope and guide physicians to treat the patient according to emergency measures. The patient must be withdrawn and be followed up until he/she recuperates. In this case, the chief investigator should report the case to hospital ethical committee and drug administration agency. The broken code and reasons for unblinding should be recorded on CRF. Meanwhile, severe adverse event report form (SAERF) will also be completed. During the revealing, allocation information must not be disclosed to the patient and any third party.

\section{Sample size}

The latest edition of Provisions for Drug Registration issued by CFDA recommends a minimum number of 300 for subjects enrolled in the trial group in a phase III clinical trial. Sample size calculation of this trial results in 24 patients in total for part A (18 in the QFGKG group and 6 in the placebo group) and 56 patients in total for part B (42 in the QFGKG group and 14 in the placebo group), respectively. Obviously, the calculated patient number in the QFGKG group is much smaller than the recommended one. Given the above, we set the sample size according to the Provisions for Drug Registration. 480 patients in total would be included, with 360 being assigned to the QFGKG group and 120 being assigned to the placebo group, considering a maximal dropout rate of $20 \%$. Calculation assumed an $80 \%$ power for a two-sided log-rank test at a $5 \%$ significance level.

\section{Interventions}

After enrollment, eligible patients will be randomly assigned to receive twice daily QFGKG $12.0 \mathrm{~g}$ or twice daily dummy QFGKG (placebo) $12.0 \mathrm{~g}$. The use of placebo as a comparator in this trial largely depends on empirical evidence $[17,28,29]$ and Guidelines for Clinical Research of New Chinese Medicine [26]. Besides, the absence of generally recognized therapy and self-healing tendency of PIC also support the use of placebo. QFGKG and placebo in this trial are manufactured by Baotou TCM Co., Ltd. The Latin names, English names and Chinese Pinyin of the four herbs used in QFGKG are listed in Table 6. Both withingroup changes and between-group changes are assessed across three time-points during treatment. As to the treatment duration, a longer (14 days) treatment in part $\mathrm{A}$ is conducted for the sake of more cured cases, while a shorter treatment (10 days) in part B is enough for monitoring noticeable changes in cough symptom score. A two-day follow-up will be conducted for all patients. At the initial drug dispensing stage, patients are advised to strictly follow protocol instructions, which will be reiterated at each study visit. Participants are requested to genuinely report their daily dosage of drugs that have been actually taken and to return unused ones to physicians at each study visit. Drug using and returning will be recorded on CRF by physicians. Unused drugs will be finally collected and counted by drug managers and then be destroyed by the sponsor at the end of the trial. All previous and concomitant treatments, including drug and non-drug therapies, taken one week before and during the intervention will be recorded on CRF. Concomitant treatment for comorbidities such as hypertension, diabetes and other chronic conditions are permitted during the intervention. Whereas, the use of antibiotics, Western or other antitussives, and non-drug cough suppressing therapies such as acupuncture and cupping are strongly discouraged to prevent potential systemic effects. Participating centers are required to record dropout cases and reasons, and also side effects happen during the intervention.

\section{Outcome measures}

Part $A$

Primary outcome The primary outcome of part A is time to cough resolution, i.e. the time to the first day when cough completely disappears. Cough resolution is defined as both daytime cough symptom score nighttime cough symptom score graded as 0 , for $48 \mathrm{~h}$. If the total cough symptom score is classified as 0 in the two successive days, the previous day should be considered as the first day when cough completely disappears (Note: cough symptom score is used to assess cough symptoms within the last $24 \mathrm{~h}$ ). For example, if the total cough symptom score of a patient is rated as 0 on the fifth day and the

Table 6 Latin name, English name and Chinese pinyin of the four herbs

\begin{tabular}{lll}
\hline Latin name & English name & Chinese pinyin \\
\hline Ephedra sinica Stapf & Herba Ephedrae & Ma Huang \\
Sinomenium acutum & Caulis Sinomenii & Qing Feng Teng \\
Stemona japonica (Blume) Miq. & Radix Stemonae & Bai Bu \\
Aster tataricus L.f. & Aster tataricus & Zi Wan \\
\hline
\end{tabular}


sixth day after intervention, then the time to cough resolution is regarded as five days.

Secondary outcomes Secondary outcomes of interest in part A include cough symptom score, time to cough relief and TCM curative effect. Cough relief is defined as both daytime cough symptom score and nighttime cough symptom score $\leq 1$ or reduce by one score, for $48 \mathrm{~h}$. TCM curative effect is calculated as percentage of cumulative TCM symptom score reduction (PSSR) estimated between baseline and post-intervention, which is categorized into four grades, complete recovery (PSSR $\geq 95 \%$ ), excellent effect (95\% > PSSR $\geq 70 \%$ ), modest effect $(70 \%>$ PSSR $\geq 30 \%)$ and no effect (PSSR $<30 \%)$. The TCM symptom score system used in the protocol follows the Guidelines for Clinical Research of New Chinese Medicine [26], in which primary and secondary symptoms are given graded scores. The TCM symptom score and TCM signs system is provided in Fig. 2. TCM signs will also be assessed, but not scored. PSSR is calculated according to the following formula:

$\mathrm{PSSR}=\left(\frac{\text { symptom score before treatment-symptom score after treatment }}{\text { symptom score before treatment }}\right) \times 100 \%$

\section{Part $B$}

Primary outcome The primary outcome of part B is cough symptom score, which will be assessed across baseline, $6 \pm 1$ days into the intervention, post-intervention (11 \pm 1 days) and 2 days post-intervention if necessary. Simplified cough symptom score (SCSS) recommended by Chinese Medical Association [30] is developed based on the Asthma and Cough Symptom Score Estimation Method [31]. It consists of daytime score and nighttime score, ranging from 0 to 3, respectively. The cough symptom score scale is provided in Table 7. SCSS, which is usually used to determine the frequency and diurnal variation of cough, and also to reflect the quality of life, has been verified as reproducible and well-responsive to treatments [32]. Daytime is arbitrarily defined as 8 a.m. to 8 p.m. and nighttime as 8 p.m. to 8 a.m.

Secondary outcomes Secondary outcomes of part B include visual analogue scale (VAS) [33], cough recovery rate, time to cough resolution, time to cough relief, TCM curative effect and cough-specific quality of life questionnaire (CQLQ) score (Chinese version). The reliability, validity and responsiveness of the Chinese version of CQLQ had been approved [21]. The production and development of this version were conducted followed the international standard guideline [34] through the comprehensive processes of translation, back-translation, committee review and pre-testing technique use. Professor Irwin, the author of the original CQLQ, granted the permission to produce the Chinese version [21].

\section{Safety outcomes}

Safety outcomes in this trial include blood routine test, urine routine test, stool routine test, electrocardiogram, liver function test (ALT, AST, STB, ALP, and $\gamma$-GT) and renal function test (Bun and $\mathrm{Cr}$ ). These biological parameters will be monitored both before and after trial intervention. Urine pregnant test (UPT) is supposed to be done before enrolling a female patient of childbearing age.

\section{Data management}

Completed CRFs will be reviewed by both chief investigators and a qualified research monitor delegated by

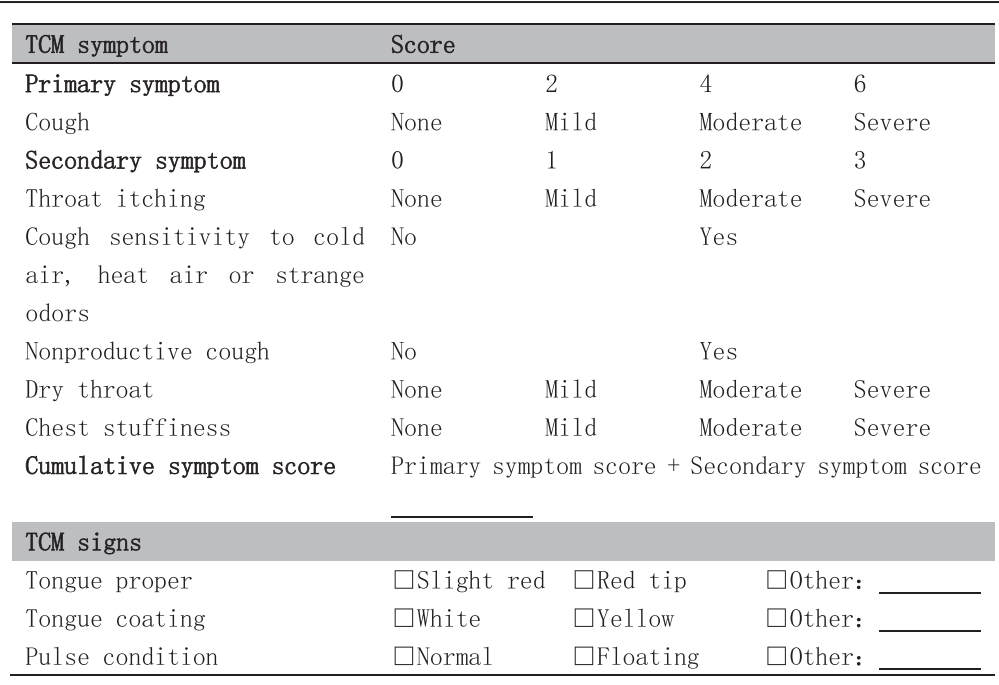

Fig. 2 TCM symptom score and TCM signs 
Table 7 Cough symptom score scale

\begin{tabular}{|c|c|c|}
\hline Score & $\begin{array}{l}\text { Daytime cough symptom } \\
\text { score }\end{array}$ & $\begin{array}{l}\text { Nighttime cough symptom } \\
\text { score }\end{array}$ \\
\hline 0 & no cough during the day & no cough during the night \\
\hline 1 & $\begin{array}{l}\text { occasional cough for short } \\
\text { periods }\end{array}$ & $\begin{array}{l}\text { cough for short periods } \\
\text { before sleep or occasional } \\
\text { cough during the night }\end{array}$ \\
\hline 2 & $\begin{array}{l}\text { frequent coughing, which } \\
\text { did slightly interfere with } \\
\text { usual daytime activities }\end{array}$ & $\begin{array}{l}\text { frequent coughing, which } \\
\text { did slightly interfere with } \\
\text { sleep }\end{array}$ \\
\hline 3 & $\begin{array}{l}\text { distressing coughs, which } \\
\text { did seriously interfere with } \\
\text { usual daytime activities }\end{array}$ & $\begin{array}{l}\text { distressing coughs preventing } \\
\text { any sleep }\end{array}$ \\
\hline
\end{tabular}

Beijing Qihuang Medicine Clinical Research Center. CRFs will be delivered for data management. All study data will be entered in computerized database using EpiData 3.1 software by two data mangers independently, and results will be cross checked for accuracy and consistency. Data entered into the database can be accessible only through authorized identification code and password. Moreover, missing data or false data, if there is any, will be detected by designed application program and recorded by data managers, who are qualified to recheck the original CRFs to ensure the correctness, completeness, and consistency of entered data. The database would be locked after data input. Original CRFs will be kept on file in a secure manner at individual participating sites by chief investigators.

\section{Statistical analysis}

Beijing Qihuang Medicine Clinical Research Center is in charge of data statistical analysis. The statistical analysis will be masked. A group of professional statisticians who are independent of all the other process of the study would perform the statistical analysis. Full Analysis Set (FAS) would be applied for all participants who have been randomly allocated and have taken QFGKG or placebo. Non-completers who never use any test drugs will be excluded. Missing values of primary variations will be imputed using the last observation carried forward (LOCF) approach according to intention-to-treat (ITT) principle. Cases in Per Protocol Set (PPS) are those who thoroughly adhere to the protocol without absence of baseline characteristics. Analysis of primary outcome and curative effect will be carried out using FAS approach and PPS approach. Safety Analysis Set (SS) includes all randomized patients who have accomplished at least one study visit. Participating centers are required to sum up participants number in each set and list participants who are removed from PPS. The Chi-square test or Fisher's exact test will be performed for the categorical variables, and the Student's t-test will be used for continuous normally distributed variables. For data that is not normally distributed, intra-group or inter-group differences before and after treatment will be analyzed by
Wilcoxon rank sum test. The proportion of patients with adverse events in two groups will be compared using the chi-square test or Fisher's exact test. The statistical significance level is set at $P<0.05$ and relative risk (RR) with corresponding $95 \%$ confidence interval $(\mathrm{CI})$ to compare dichotomous variables will be calculated. Table 8 shows the method of analysis for specific outcomes.

\section{Ethics}

This trial is conducted in accordance with the ethical and scientific principles that originate from the Declaration of Helsinki and GCP [35] and local regulations (http://www.sda.gov.cn/ypzcgl/ml.htm). The trial has been authorized by CFDA (Clinical Trial Approval No. 2010 L00279). The current protocol and written informed consent have been approved by the Ethics Committee of Clinical Trials and Biomedicine of West China Hospital of Sichuan University. Until the required quota of participants is reached, all potential patients can join in this trial through two ways, advertisement and review of health records by respiratory physicians. The recruitment advertisement has also been approved by the ethics committee. Participants are promised to be paid 200 $\mathrm{RMB}$ as financial incentive for enrollment when the whole trial is finished, and they will be informed detailed information about this trial regarding objectives, procedure, potential risks and benefits, and their rights and obligations prior to enrollment. An informed consent that is written in easy-to-understand language will be given to participants. Patients' full understanding of the protocol and contentment to every response from physicians must be ensured. Interventions will not be initiated until the written informed consent is obtained from all participants. Physicians are required to give their contact details to each patient recruited. As regards secrecy and confidentiality, data of every single patient will be recorded anonymously, and any information that is suspected to reveal patients' identification will be concealed. Patients' confidentiality will be preserved at all times during the whole trial and their medical records will definitely be properly archived in National Drug Clinical Trial Institution, not disclosed to any third party.

\section{Discussion}

Postinfectious cough, a common but not widely recognized and understood disease, imposes a great burden on both physical and mental health of patients. Exploring an effective treatment for PIC is an urgent necessity. TCM is a structured intervention that has shown some promise in the management of persistent cough. A growing number of clinical trials are studying the effect of TCM on PIC. But unfortunately, existing unconvincing evidence makes it awkward to interpret findings in a consistent manner or 
Table 8 Outcomes and methods of analysis

\begin{tabular}{|c|c|c|c|}
\hline Outcome/variable & Hypothesis & Measures & Methods of analysis \\
\hline \multirow[t]{2}{*}{ Baseline balance test } & & $\begin{array}{l}\text { Quantitative outcomes (age, disease duration, } \\
\text { body temperature, heart/respiratory rate, blood } \\
\text { pressure) }\end{array}$ & T-test/Wilcoxon rank sum test \\
\hline & & $\begin{array}{l}\text { Qualitative outcomes (gender, marriage, race, } \\
\text { previous treatment) }\end{array}$ & $\begin{array}{l}\text { Chi-squared test/Fisher exact test/rank-sum } \\
\text { test }\end{array}$ \\
\hline $\begin{array}{l}\text { Adherence at post- } \\
\text { intervention }\end{array}$ & & $\begin{array}{l}\text { Percent and cases of adherence in previous 10/ } \\
14 \\
\text { days }<80 \%,>120 \% \text { and } 80 \%-120 \%\end{array}$ & Chi-squared test/Fisher exact test \\
\hline Concomitant treatments & & Percent and cases of concomitant treatments & Chi-squared test/Fisher exact test \\
\hline Intervention duration & & $\begin{array}{l}\text { Formula: the last day using test drugs-the first } \\
\text { day using test drugs }+1\end{array}$ & T-test/Wilcoxon rank sum test \\
\hline \multicolumn{4}{|l|}{ Primary } \\
\hline Time to cough resolution & $\begin{array}{l}\text { improvement } \\
\text { occurred }\end{array}$ & & Kaplan-Meier method/Log-rank test \\
\hline Cough symptom score & $\begin{array}{l}\text { improvement } \\
\text { occurred }\end{array}$ & & T-test/Wilcoxon rank sum test \\
\hline \multicolumn{4}{|l|}{ Secondary } \\
\hline VAS score & $\begin{array}{l}\text { improvement } \\
\text { occurred }\end{array}$ & & T-test/Wilcoxon rank sum test \\
\hline TCM symptom score & $\begin{array}{l}\text { improvement } \\
\text { occurred }\end{array}$ & & T-test/Wilcoxon rank sum test \\
\hline TCM curative effect & $\begin{array}{l}\text { improvement } \\
\text { occurred }\end{array}$ & $\begin{array}{l}\text { Percent and cases of four grades: complete } \\
\text { recovery, excellent effect, modest effect and } \\
\text { no effect }\end{array}$ & Cochran-Mantel-Haenszel Chi-squared test \\
\hline CQLQ score & $\begin{array}{l}\text { improvement } \\
\text { occurred }\end{array}$ & Questionnaire & T-test/Wilcoxon rank sum test \\
\hline Cough disappearance rate & $\begin{array}{l}\text { improvement } \\
\text { occurred }\end{array}$ & & $\mathrm{CMH}$ Chi-squared test \\
\hline Time to cough relief & $\begin{array}{l}\text { improvement } \\
\text { occurred }\end{array}$ & & Kaplan-Meier method/Log-rank test \\
\hline
\end{tabular}

confirm a conclusion. Although our previous phase II trial is the first study using TCM to treat PIC, some limitations still exist [20], such as the absence of follow-up. This trial aims to fill this gap by undertaking a well-designed multicentre, placebo-controlled, double-blind randomized trial to evaluate the efficacy and safety of a TCM formula, Qing-Feng-Gan-Ke-Granules. Moreover, this trial will be an outstanding supplement to this area by providing tangible benefits for PIC research.

In this trial, we conduct two simultaneous study parts that are mutually independent but not mutually exclusive. We aim to not only propose a new effective remedy for PIC but also practice a novel methodology for clinical trials, that is "one study, one primary outcome". As we all know, a well designed clinical trial is always expected to answer multiple main clinical questions. Nevertheless, sometimes it is unreasonable to assess multi-primary outcomes in one study. In this trial, we intend to investigate the effectiveness of QFGKG on different clinical outcomes. In this case, it may be more convincing and persuasive to study different groups of patients and conduct different intervention durations to achieve exclusive primary outcome by separating the original trial into study parts. We believe that if considering the results of the two study parts as a whole, it would be helpful not only for figuring out ongoing objectives but also for clarifying further implications. Therefore, we assume that this novel design contributes to maximized efficacy, lower cost, a better risk-benefit ratio of treatment and more convincing results with strong application value and may be recommended for further clinical intervention trials. This is the most all-important distinguishing feature of this trial. Another breakthrough in this protocol is that we highlight the importance of two clinical related outcomes, time to cough resolution and quality of life, which are seldom assessed in previous studies [14].

Several limitations and drawbacks of this study are as follows. Firstly, since we are going to conduct different intervention durations in the two parts, some patients with relatively mild cough would be treated for 14 days while those with relatively severe cough will be treated for 10 days, which is paradoxical and against common clinical practice. Secondly, although a careful knowledge of medical history 
and physical examination including serologic tests may provide clues to diagnosis, the diagnosis of PIC still largely depends on clinical exclusion [5]. Therefore, the possibility of misdiagnosis cannot be ruled out. Thirdly, an objective cough measurement is not included in this study. Many video and sound recording surveillance have been used in clinic to objectively monitor the cough frequency and severity [36-39]. However some studies reported a poor relationship between cough frequency and cough symptom score [37-39]. Furthermore, although operating a manual recording instrument is easy for patients, it requires a good compliance, otherwise, considerable errors could be induced. In addition, how to effectively and sensitively distinguish and filter the external vocal interference, and also record the weak electromyographic signals of pulmonary muscles are still the technical bottlenecks for the development of $24 \mathrm{~h}$ cough monitors. Besides, the expensive cost also limits the use of cough recording surveillance. For the above reasons, objective cough measurements are not included in our study. Fourthly, the lack of data from old people over the age of 65 fails to provide information about the efficacy and safety of QFGKG in this group of patients.

\section{Trial status}

The recruitment process was initiated from May 01, 2013 and until now, a total of 318 participants have been included.

\section{Abbreviations \\ ALP: Alkaline Phosphatase; ALT: Alanine aminotransferase; AST: Aspartate aminotransferase; BPT: bronchial provocation test; BUN: Blood urea nitrogen; $\mathrm{CMH}$ : Cochran-Mantel-Haenszel; CQLQ: cough-related quality of life questionnaire; Cr: Creatinine; CR: chest radiography; CRF: case report form; ECG: Electrocardiogram; FAS: Full Analysis Set; GCP: good clinical practice; QFGKG: Qing-Feng-Gan-Ke Granules; CFDA: China Food and Drug Administration; SS: Safety Analysis Set; PPS: Per Protocol Set; TCM: traditional Chinese medicine; UPT: urine pregnant test; URTI: upper respiratory tract inflammation; $\gamma$-GT: $\gamma$-Glutamyl Transpeptidase.}

\section{Competing interests}

The authors declare that they have no competing interests.

\section{Authors' contributions}

WL and HL-J are co-first authors, they contributed equally to this work. BM contributed to the initiation of the study design, funding management, and to contract with individual centers. WL, HL-J and RM-Z contributed to review and verify this protocol. $W L, H L-J, B M, Y Q-Z$ and $R M-Z$ contributed to refinement and implementation of the protocol. LY-C, SY-L, LJ-L, YY-L, FG-J and $\mathrm{BM}$ are identified as chief investigators and contributed to arrangement of clinical process at individual centers. WL contributed to the draft of manuscript. Agreement of final version had been obtained from all authors.

\section{Acknowledgement}

Expenses of whole process of this trial are funded by the Beijing Qihuang Medicine Clinical Research Center (the sponsor), an independent Contract Research Organization (CRO). The expenses cover relevant meeting costs, central organizational costs, local organizational costs, statistical analysis costs, patient financial incentive, intervention drugs, equipment and consumables, clinical examination costs, and unforeseeable expenses such as compensations for participants who suffer severe adverse events and even legal fee.

\section{Author details}

1 Department of Integrated Traditional and Western Medicine, West China Hospital of Sichuan University, 37 Guoxue Lane, Chengdu, Sichuan Province 610041, China. ${ }^{2}$ Department of Respiratory and Critical Care Medicine, Tangdu Hospital of The Fourth Military Medical University, 1 Xinsi Road, Xi'an, Shaanxi Province 710038 , China. ${ }^{3}$ Department of Respiratory Medicine, Affiliated Hospital of Jiangxi University of Traditional Chinese Medicine, 445 Bayi Avenue, Nanchang, Jiangxi Province 330006, China. ${ }^{4}$ Department of Respiratory Medicine, The Second Affiliated Hospital of Tianjin University of Traditional Chinese Medicine, 816 Zhenli Street, Tianjin 300150, China. ${ }^{5}$ Department of Respiratory Medicine, Affiliated Hospital of Inner Mongolia Medical University, 1 North Tongdao Street, Hohhot, Inner Mongolia 010000, China. ${ }^{6}$ Department of Respiratory Diseases, The First Affiliated Hospital of Henan University of Traditional Chinese Medicine, 19 Renmin Road, Zhengzhou, Henan Province 450000, China. ${ }^{7}$ Department of Respiratory Medicine, The First Affiliated Hospital of Guangxi University of Traditional Chinese Medicine, 89-9 Dongge Road, Nanning, Guangxi Province 530023, China.

Received: 24 June 2014 Accepted: 10 August 2015

Published online: 19 August 2015

\section{References}

1. Chung KF, Pavord ID. Prevalence, pathogenesis, and causes of chronic cough. Lancet. 2008;371:1364-74.

2. Kwon NH, Oh MJ, Min TH, Lee BJ, Choi DC. Causes and clinical features of subacute cough. Chest. 2006;129:1142-7.

3. Zimmerman B, Silverman FS, Tarlo SM, Chapman KR, Kubay JM, Urch B. Induced sputum: comparison of postinfectious cough with allergic asthma in children. J Allergy Clin Immunol. 2000;105:495-9.

4. Irwin RS, Baumann MH, Bolser DC, Boulet LP, Braman SS, Brightling CE, et al. American College of Chest Physicians (ACCP): Diagnosis and management of cough executive summary: ACCP evidence-based clinical practice guidelines. Chest. 2006;129:1S-23S.

5. Braman SS. Postinfectious cough: ACCP evidence-based clinical practice guidelines. Chest. 2006;129:138S-46S.

6. Raeessi MA. Aslani J, Raeessi N, Gharaie H, Karimi Zarchi AA, Raeessi F. Honey plus coffee versus systemic steroid in the treatment of persistent post-infectious cough: a randomised controlled trial. Prim Care Respir J. 2013;22:325-30.

7. Ishida T, Yokoyama T, Iwasaku M, Saigusa M, Fukuyama H, Nakagawa $H_{\text {, }}$ et al. Clinical investigation of postinfectious cough among adult patients with prolonged cough. Nihon Kokyuki Gakkai Zasshi. 2010;48:179-85.

8. Nicole MR, Peter GG. Extrathoracic airway hyperresensiveness as a mechanism of post infectious cough: case report. Cough. 2008; doi:10.1186/1745-9974-4-7

9. Saleh H. Rhinosinusitis, laryngopharyngeal reflux and cough: an ENT viewpoint. Pulm Phpartacol Ther. 2009;22:127-9.

10. Cho YS, Lee CK, Yoo B, Moon HB. Cough sensitivity and extrathoracic airway responsivenesss to inhaled capsaicin in chronic cough patients. J Korean Med Sci. 2002;17:616-20.

11. Wang K, Birring SS, Taylor K, Fry NK, Hay AD, Moore M, et al. Montelukast for postinfectious cough in adults: a double-blind randomised placebocontrolled trial. Lancet Respir Med. 2014;2:35-43.

12. Morice AH, McGarvey L, Pavord I, British Thoracic Society Cough Guideline Committee. Recommendations for the management of cough in adults. Thorax. 2006;61:11-24.

13. Raeessi MA, Aslani J, Gharaie H, Karimi Zarchi AA, Raeessi N, Assari S. Honey with Coffee: A new finding in the treatment of persistent postinfectious cough. Iran J OtoRhino-Laryng. 2011;23:1-8.

14. Anderson-James S, Marchant JM, Acworth JP, Turner C, Chang AB. Inhaled corticosteroids for subacute and chronic cough in children. Cochrane Database Syst Rev. 2013; doi:10.1002/14651858.CD008888.pub2

15. Ziaee V, Akbari Hamed E, Hoshmand A, Amini H, Kebriaeizadeh A, Saman K. Side effects of dextromethorphan abuse, a case series. Addict Behav. 2005:30:1607-13.

16. Rossi S. Australian Medicines Handbook. Adelaide: The Australian Medicines Handbook Unit Trust; 2013

17. Liu W, Jiang HL, Mao B. Chinese Herbal Medicine for Postinfectious Cough: A Systematic Review of Randomized Controlled Trials. Evid Based Complement Alternat Med. 2013; doi:10.1155/2013/906765 
18. Ma JL. Clinical research for effectiveness of Qu-Feng-Xuan-Fei granule in the management of patients with post-infectious cough. Beijing University of Chinese Medicine. 2006

19. Ma JL, Shi LQ, Xi N. Analysis and study on 60 cases of postinfectious cough treated with eliminating wind and releasing lung medicines. J Beijing Univ Tradit Chin Med. 2007;30:67-9.

20. Jiang HL, Mao B, Wang L, Zhang RM, She B, Jin FG, et al. Chinese herbal formula in the treatment of post-infectious cough: a multicenter, randomized, double-blind, placebo-controlled trial. Chinese Medicine. 2015. In press.

21. Ma HM, Chen QD, Liu XY, Wu Y, Liu SM. Reliability and validity and responsiveness evaluation of the Chinese version of cough-specific qualityof-life questionnaire. Chin J Pract Intern Med. 2013;33:483-75.

22. Chan AW, Tetzlaff JM, Gøtzsche PC, Altman DG, Mann H, Berlin JA, et al. SPIRIT 2013 explanation and elaboration: guidance for protocols of clinical trials. BMJ. 2013;346:e7586.

23. Schulz K, Altman D, Moher D. CONSORT Group. CONSORT 2010 Statement: updated guidelines for reporting parallel group randomised trials. BMC Med. 2010;8:18.

24. Chan AW, Tetzlaff JM, Altman DG, Laupacis A, Gøtzsche PC, Krleža-Jerić K, et al. SPIRIT 2013 statement: defining standard protocol items for clinical trials. Ann Intern Med. 2013;158:200-7.

25. Asthma Study Group, Committee of Respiratory Disease, Chinese Medical Association. "Guideline: diagnosis and management of cough (version 2009)". Chin J Tuberc Respir Dis. 2009:32:407-13.

26. Zhen XY. Guidelines for Clinical Research of New Chinese Medicine. Beijing: China Medical Science Press; 2002.

27. State Administration of Traditional Chinese Medicine. Standards of Traditional Chinese Syndrome Diagnosis and Treatment. Beijing, China: China Press of Traditional Chinese Medicine; 1994.

28. Fu JJ, Wang G, Mao B. The assessment of scientificness and ethic of placebo controlled trials in traditional Chinese medicine. Chin J Evid Based Med. 2008:8:781-5.

29. Zhong YQ, Fu JJ, Liu XM, Diao X, Mao B, Fan T, et al. The reporting quality, scientific rigor, and ethics of randomized placebo-controlled trials of traditional Chinese medicine compound formulations and the differences between Chinese and non-Chinese trials. Curr Ther Res. 2010;71:30-49.

30. Asthma Study Group, Committee of Respiratory Disease, Chinese Medical Association. "Guideline: diagnosis and management of cough.". Chin J Gen Pract. 2009:8:608-13.

31. Hsu JY, Stone RA, Logan-Sinclair RB, Worsdell M, Busst CM, Chung KF. Coughing frequency in patients with persistent cough: assessment using a 24 h ambulatory recorder. Eur Respir J. 1994;7:1246-53.

32. Zhao T, Qiu ZH, Wang L, Yu L, Lv HJ, Qiu ZM. Validation of the reliability and clinical value of the simplified cough score. Chin J Gen Pract. 2012;11:273-6

33. Raj AA, Birring SS. Clinical assessment of chronic cough severity. Pulm Pharmacol Ther. 2007;20:334-7.

34. Guillemin F, Bombardier C, Beaton D. Cross-cultural adaptation of health-related quality of life measures: literature review and proposed guidelines. J Clin Epidemiol. 1993:46:1417-32.

35. International Conference on Harmonisation of Technical Requirements for Registration of Pharmaceuticals for Human Use $(\mathrm{ICH})$ adopts Consolidated Guideline on Good Clinical Practice in the Conduct of Clinical Trials on Medicinal Products for Human Use. Int Dig Health Legis. 1997; 48:231-234.

36. Hsu JY, Stone RA, Logan-Sinclair RB, Worsdell M, Busst CM, Chung KF. Coughing frequency in patients with persistent cough:assessment using a 24-h ambulatory recorder. Eur Resp J. 1994;7:1246-53.

37. Chang AB, Phelan PD, Robertson CF, Newman RG, Sawyer SM. Frequency and perception of cough severity. J Paediatr Child Health. 2001;37:142-5.

38. Chung KF. Assessment and Measurement of cough:the value of new tools. Pulm Pharmacol Ther. 2002;15:267-72.

39. Chang $A B$, Newman RG, Carlin JB. Subjective scoring of cough in children:parent-completed vs. child-completed diary cards vs. an objective method. Eur Respir J. 1998:11:462-6.

\section{Submit your next manuscript to BioMed Central and take full advantage of:}

- Convenient online submission

- Thorough peer review

- No space constraints or color figure charges

- Immediate publication on acceptance

- Inclusion in PubMed, CAS, Scopus and Google Scholar

- Research which is freely available for redistribution

Submit your manuscript at www.biomedcentral.com/submit 